Louisiana State University

LSU Digital Commons

$4-11-2018$

\title{
The use of cosmic-ray muons in the energy calibration of the Beta-decay Paul Trap silicon-detector array
}

\author{
T. Y. Hirsh \\ University of Manitoba
}

A. Pérez Gálvan

Argonne National Laboratory

M. T. Burkey

Argonne National Laboratory

A. Aprahamian

University of Notre Dame

F. Buchinger

Université McGill

See next page for additional authors

Follow this and additional works at: https://digitalcommons.Isu.edu/physics_astronomy_pubs

\section{Recommended Citation}

Hirsh, T., Pérez Gálvan, A., Burkey, M., Aprahamian, A., Buchinger, F., Caldwell, S., Clark, J., Gallant, A., Heckmaier, E., Levand, A., Marley, S., Morgan, G., Nystrom, A., Orford, R., Savard, G., Scielzo, N., Segel, R., Sharma, K., Siegl, K., \& Wang, B. (2018). The use of cosmic-ray muons in the energy calibration of the Beta-decay Paul Trap silicon-detector array. Nuclear Instruments and Methods in Physics Research, Section A: Accelerators, Spectrometers, Detectors and Associated Equipment, 887, 122-127.

https://doi.org/10.1016/j.nima.2018.01.021

This Article is brought to you for free and open access by the Department of Physics \& Astronomy at LSU Digital Commons. It has been accepted for inclusion in Faculty Publications by an authorized administrator of LSU Digital Commons. For more information, please contact ir@lsu.edu. 


\section{Authors}

T. Y. Hirsh, A. Pérez Gálvan, M. T. Burkey, A. Aprahamian, F. Buchinger, S. Caldwell, J. A. Clark, A. T. Gallant, E. Heckmaier, A. F. Levand, S. T. Marley, G. E. Morgan, A. Nystrom, R. Orford, G. Savard, N. D. Scielzo, R. Segel, K. S. Sharma, K. Siegl, and B. S. Wang 
The use of cosmic-ray muons in the energy calibration of the Beta-decay Paul Trap silicon-detector array

T. Y. Hirsh, A. Perez Galvin, M. Burkey, A. Aprahamian, F. Buchinger, S. Caldwell, J. A. Clark, A. Gallant, E. Heckmaier, A. F. Levand, S. T. Marley, G. E. Morgan, A. Nystrom, R. Orford, G. Savard, N. D. Scielzo, R. Segel, K. S. Sharma, K. Siegl, B. S. Wang

June 8, 2017

Nuclear Instruments and Methods in Physics Research A 
This document was prepared as an account of work sponsored by an agency of the United States government. Neither the United States government nor Lawrence Livermore National Security, LLC, nor any of their employees makes any warranty, expressed or implied, or assumes any legal liability or responsibility for the accuracy, completeness, or usefulness of any information, apparatus, product, or process disclosed, or represents that its use would not infringe privately owned rights. Reference herein to any specific commercial product, process, or service by trade name, trademark, manufacturer, or otherwise does not necessarily constitute or imply its endorsement, recommendation, or favoring by the United States government or Lawrence Livermore National Security, LLC. The views and opinions of authors expressed herein do not necessarily state or reflect those of the United States government or Lawrence Livermore National Security, LLC, and shall not be used for advertising or product endorsement purposes. 


\title{
The use of cosmic-ray muons in the energy calibration of the Beta-decay Paul Trap silicon-detector array
}

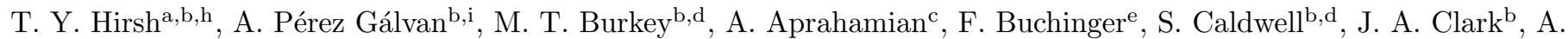 \\ T. Gallant' ${ }^{\mathrm{g}}$ E. Heckmaier ${ }^{\mathrm{j}}$, A. F. Levand ${ }^{\mathrm{b}}$, S. T. Marley ${ }^{\mathrm{c}}$, G. E. Morgan ${ }^{\mathrm{a}, \mathrm{b}}$, A. Nystrom ${ }^{\mathrm{b}, \mathrm{c}}$, R. Orford ${ }^{\mathrm{b}, \mathrm{e}}$, G. Savard $^{\mathrm{b}, \mathrm{d}}$,

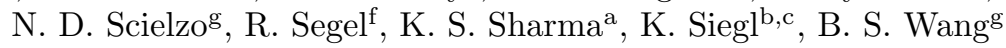 \\ ${ }^{a}$ Department of Physics and Astronomy, University of Manitoba, Winnipeg, Manitoba R3T 2N2, Canada \\ ${ }^{b}$ Physics Division, Argonne National Laboratory, Argonne, Illinois 60439, USA \\ ${ }^{c}$ Department of Physics, University of Notre Dame, Notre Dame, Inidiana 46556, USA \\ ${ }^{d}$ Department of Physics, University of Chicago, Chicago, Illinois 60637, USA \\ ${ }^{e}$ Department of Physics, McGill University, Montréal, Québec H3A 2T8, Canada \\ ${ }^{f}$ Department of Physics and Astronomy, Northwestern University, Evanston, Illinois 60208, USA \\ ${ }^{g}$ Nuclear and Chemical Sciences Division, Lawrence Livermore National Laboratory, Livermore, California 94550, USA \\ ${ }^{h}$ Soreq NRC, Yavne 81800, Israel \\ ${ }^{i}$ Vertex Pharmaceuticals, 11010 Torreyana Road, San Diego, CA 92121, USA \\ ${ }^{j}$ Department of Physics and Astronomy, University of California, Irvine, California 92697-4575, USA
}

\begin{abstract}
This article presents an approach to calibrate the energy response of double-sided silicon strip detectors (DSSDs) for low-energy nuclear-science experiments by utilizing cosmic-ray muons. For the 1-mm-thick detectors used with the Beta-decay Paul Trap, the minimum-ionizing peak from these muons provides a stable and time-independent in situ calibration point at around $300 \mathrm{keV}$, which supplements the calibration data obtained above $3 \mathrm{MeV}$ from $\alpha$ sources. The muon-data calibration is achieved by comparing experimental spectra with detailed Monte Carlo simulations performed using GEANT4 and CRY codes. This additional information constrains the calibration at lower energies, resulting in improvements in quality and accuracy.
\end{abstract}

\section{Introduction}

Detailed studies of the $\beta$-delayed $\alpha$-particle emission in the decay of ${ }^{8} \mathrm{~B}$ and ${ }^{8} \mathrm{Li}$ are important for solar-neutrino spectroscopy [1, 2, 3, 4, 5] and low-energy searches for physics beyond the Standard Model [6, 7]. The $\alpha$-particle energy spectrum resulting from the break up of the broad excited state in the ${ }^{8}$ Be daughter nucleus peaks at around $1.5 \mathrm{MeV}$ and extends up to about $8 \mathrm{MeV}$. In these experiments, silicon detectors are used to determine the energy of the emitted $\alpha$ particles and spectroscopy-grade $\alpha$ sources can provide high-precision calibrations, at energies above $3 \mathrm{MeV}$.

However, characterizing the detector response below 3 $\mathrm{MeV}$, where the bulk of the $\alpha$-particle energy spectrum lies, is more challenging because no spectroscopy-grade $\alpha$ sources are available. Previous experiments [2, 4, 5] have relied on $\beta$-delayed $\alpha$ emission lines at 2.15 and $4.43 \mathrm{MeV}$ from the decay of ${ }^{20} \mathrm{Na}$ to ${ }^{20} \mathrm{Ne}$ to extend the calibration to lower energies. However, because ${ }^{20} \mathrm{Na}$ has a half-life of only $447.9(23) \mathrm{ms}$, this calibration requires access to accelerator beam time and therefore is usually limited in availability and statistics.

In this article, a method is presented that utilizes the spatial and spectral information provided by the ever-present cosmic-ray muon flux passing through the pairs of position- sensitive double-sided silicon strip detectors (DSSDs) to add an additional calibration point below $1 \mathrm{MeV}$. The muon minimum-ionizing peak provides a stable low-energy feature present both off-line and in situ during on-line experiments which can be used to supplement the calibration provided by $\alpha$-sources in the $3-8 \mathrm{MeV}$ region and anchor it at low energies. As long as the muon signature is above the detection threshold, this additional information can be used to improve the detector characterization and help monitor the stability of the detector performance. Although the use of high-energy muon interactions to calibrate the energy response of silicon detectors is a common practice in particle-physics experiments 8], to the best of our knowledge, this is the first time that this method has been applied to detectors used for low-energy nuclearphysics research.

The energy calibration is demonstrated with the DSSD detector array used with the Beta-decay Paul Trap (BPT) 9. 17. The BPT has been used to study the $\beta$ decays of ${ }^{8} \mathrm{Li}$ [6, 7] and ${ }^{8} \mathrm{~B}$, as well as $\beta$-delayed neutron emission of fission fragments [10, 11]. The experimental setup, simulations, data analysis, and impact the additional information from the muons has on the accuracy of the energy calibration are discussed below. 


\section{Experimental setup}

The BPT is a linear Paul trap which has been specially designed with thin segmented planar electrodes to allow radiation detectors to cover a large solid angle for detecting particles emitted from the trap center. A detailed description of this ion trap can be found in Refs. [9, 7] and therefore only a brief summary is provided here. A cross-sectional view of the BPT instrumented with the four DSSDs used in this work is illustrated in Fig. 1. Along the axial direction of the trap, ions are confined by electrostatic potentials applied on the three segments of each electrode. In the radial direction, the ions are confined by radiofrequency ( $\mathrm{RF}$ ) fields, arising from time-varying potentials, applied to the electrodes. The detector array consists of four identical DSSDs, each 1-mm thick with an active area of $64 \times 64 \mathrm{~mm}^{2}$, mounted to the trap frame. On the front and back faces, the DSSDs are segmented into 32 2-mm-wide strips. The segmentation allows the identification of the location of the particle interaction, thus allowing the reconstruction of the momentum direction of particles emitted from the trap center. The detectors are referred to as Top, Bottom, Left, and Right, according to their position relative to the ion-trap center as viewed along the direction of the ion beam.

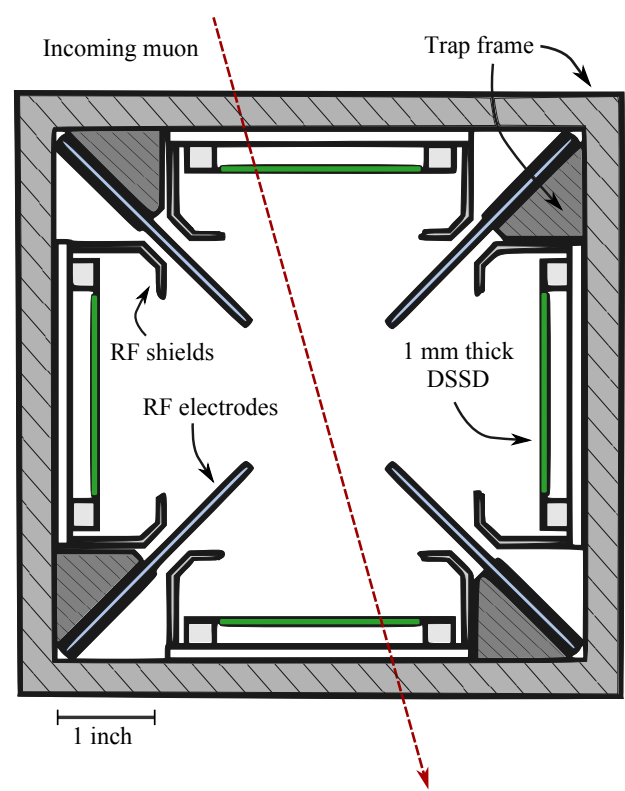

Figure 1: Cross-sectional view of the BPT. Ions are confined in the geometrical center of the trap by a combination of RF and DC fields applied to the electrodes. Four DSSDs surround the trap and are encased by a set of RF shields to minimize electrical pick-up from the trapping fields. An incoming muon which passes through two DSSDs can be identified. Alpha calibration sources placed on the RF shields are used for continuous calibration of the DSSD energy response.

The BPT is cooled to cryogenic temperatures by flowing liquid nitrogen through the trap frame which decreases the background pressure to less than $1 \times 10^{-9}$ Torr and reduces the dark current of the DSSDs to negligible levels.
A small amount of neutral high-purity ${ }^{4} \mathrm{He}$ gas is injected into the trapping volume to cool the trapped ions through collisions. After a few milliseconds, the ion cloud converges to roughly a $1-\mathrm{mm}^{3}$ volume [12. Additional information on the performance of the trap can be found in Ref. [9].

Two sets of $\alpha$ sources $\left({ }^{148} \mathrm{Gd}\right.$ and ${ }^{244} \mathrm{Cm}$ with primary energy lines of $3182.690(24) \mathrm{keV}$ and $5804.77(5) \mathrm{keV}$, respectively) provide a continuous in situ calibration of the DSSDs throughout the data collection. In addition, a precision signal pulser is used periodically to check the linearity and stability of the electronics. A detailed energy calibration is performed for the front strips of the detectors, while the back strips are primarily used for reconstructing the particle hit position and eliminating events with incomplete charge collection based on pulse-height differences. The $\alpha$ sources provide calibration points in the $3-6$ $\mathrm{MeV}$ range; the introduction of a third calibration point below $3 \mathrm{MeV}$ is desirable to improve the precision at lower energies.

\section{Simulations}

The energy loss mechanism of muons passing through matter is well described by the Bethe-Bloch formula [13. However, due to the high-energy nature of the muon-induced reactions, the secondary recoils produced may emerge from the detector volume and leave only a fraction of their energy behind. Therefore, to calculate the energy deposition in the detectors, a detailed Monte Carlo simulation is required. In this work cosmic muon interactions were simulated using the GEANT4 simulation toolkit [14, a wellvalidated radiation transport code, especially for electromagnetic interactions [15]. The simulation geometry consists of the four DSSDs and the ion trap structure (see Fig. 1).

To generate the spatial and spectral distribution of sea-level cosmic muons, the CRY (Cosmic RaY shower) library [16] was used. Derived from full atmospheric simulations and validated against experimental data, it provides a good description of cosmic-ray showers [17, 18. The muons generated by the CRY library have a broad energy spectrum distributed around $4 \mathrm{GeV}$, uniform spatial distribution, and generally downward directionality. The code takes the altitude, latitude, and date of the measurement into account to generate realistic muon spectra. For this work, the energy spectrum at sea level was considered to be a good approximation to the experiment's site altitude of $100 \mathrm{~m}$ above sea level.

The simulated muons (which represent individual muons from cosmic-ray showers) illuminate the BPT ion-trap structure and detector array. A muon that strikes two detectors (illustrated by the red dashed line in Fig. 1) is classified as a double coincidence and the angle of the impinging muon can be determined. The comparison of the experimental measurements with simulation results are discussed in the following section. 


\section{Description of methods}

The energy calibration presented here was performed using data taken during and immediately after a measurement of trapped ${ }^{8} \mathrm{~B}$ ions performed at the Argonne Tandem Linac Accelerator System (ATLAS) facility. For simplicity and as a proof-of-concept, only the calibration of the Top detector, based on muon coincidences between the Top and Bottom detectors, is summarized here. Also, the analysis here focuses on the data collected after the run because the identification of muons is cleaner in the absence of other minimum-ionizing particles, such as $\beta^{+}$ particles in the case of ${ }^{8} \mathrm{~B}$ decay, which can deposit comparable amount of energy in the detectors. The muon peak observed during and after the online data collection were consistent.

Figure 2 shows a typical energy spectrum taken by a front-strip of the Top detector. Three features are clearly visible. On the higher-energy side of the spectrum, the broad $\alpha$ peaks from the ${ }^{148} \mathrm{Gd}$ and ${ }^{244} \mathrm{Cm}$ sources can be observed at approximately channels 1500 and 3000. The prominent peak at approximately channel 200 corresponds to cosmic-ray muons. Although cosmic-ray showers contain other types of ionizing radiation, muons deposit energy in DSSD pairs at the highest rate.

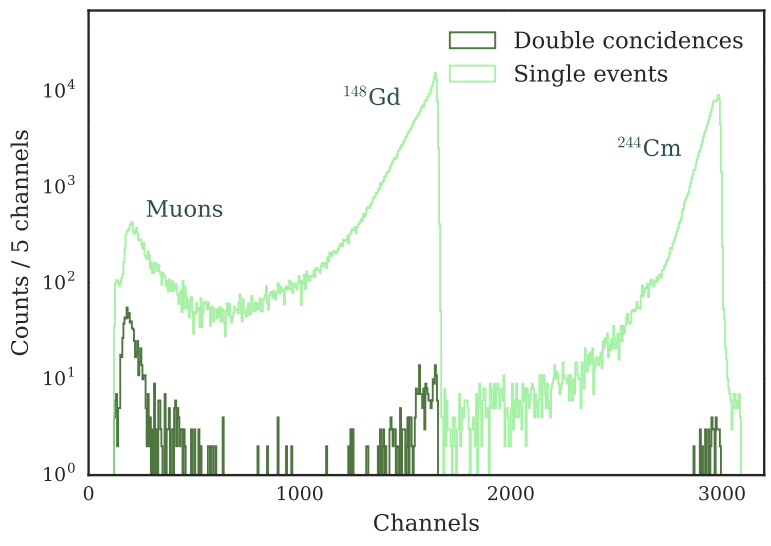

Figure 2: Energy spectrum of all events collected in a typical strip of the Top detector during the 23-hour background measurement (light curve). The three distinctive peaks correspond to alpha emission from the calibration sources and energy deposited by cosmic-ray muons. By selecting events that are in coincidence with the Bottom detector (darker curve), the muon peak is isolated and the impinging direction of muons is constrained. The remaining counts in the $\alpha$ peaks are due to accidental coincidences.

Muon events in the spectra are identified by requiring a coincidence between two opposite detectors and an energy deposition of less than $1000 \mathrm{keV}$ in each. Analysis of 23 hours of data yielded $5 \times 10^{4}$ coincidences in the Top-Bottom detectors that are identified as muon events. These events form a peak with a Landau function shape which is characterized by a skewed shape and long tail and provides a fundamental description of the energy-deposition distribution for charged particles in matter [19]. For our case the pure Landau distribution is scaled by the path length of the $\mu$ through the DSSD thickness, resulting in a slightly shifted and skewed shape in correspondence to the impinging angle. For the particular setup, the angle resolution is of the order of 1.5 degrees. The spectrum for each strip obtained in coincidence with the complete surface of the opposite detector is analyzed separately to limit the extent of this broadening.

\section{Data analysis}

A Landau function is fitted to the pulse height spectrum of each strip and the most probable value (the peak center) is calculated. Those values are compared with the most probable values provided by the equivalent Landau function fits of the GEANT4 simulation results. Figure 3 shows a comparison between experiment, simulation, and the associated fits for a typical strip in the Top detector. The Landau function fits performed using the MINUIT code, show a good agreement between simulation and experiment. A summary of the most probable values obtained for the different strips in the Top detector is shown in Fig. 4.

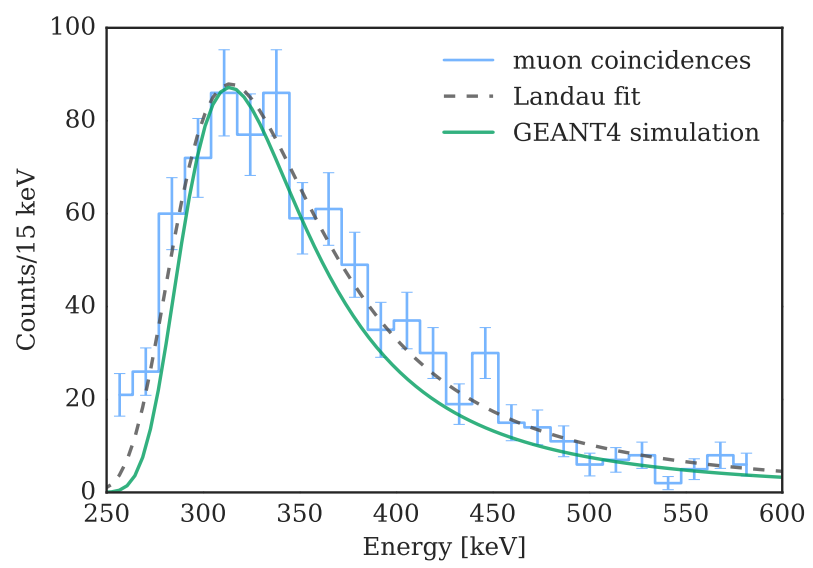

Figure 3: Spectrum of double-coincidence muon events. The curves show a comparison of the spectrum simulated to the results obtained with a typical strip of the Top detector in coincidence with any strip in the Bottom detector. The measured pulse height is calibrated using the 3-point calibration, which is described in the text.

The energy calibration is performed for each front strip individually based on the calibration points obtained from the muon signal and the two alpha sources. The alpha source spectra are fit to a function that takes into account the skewed shapes of the spectrum and the two dominant alpha-lines of ${ }^{244} \mathrm{Cm}$ to determine the peak edges. More details on this procedure are provided in Ref. [12. Due to the span of energies of interest and the different types of radiation involved, it is vitally important to correct for non-linear effects resulting from the detector response and source profile. Non-linear energy loses, which are substantial for alphas and discussed in detail in the following paragraphs, are small for the case of muons and can be safely 


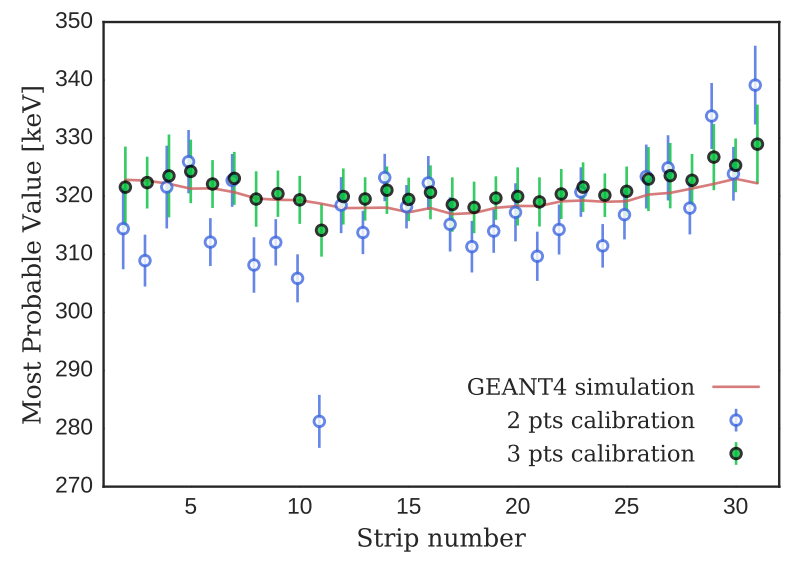

Figure 4: Most probable values of the Landau function fits for the different front strips across the Top detector. A comparison of the calibrations based on alpha sources and muons (3-points calibration) and with alpha sources alone (2-points calibration), along with the GEANT4 prediction, is shown.

neglected [13. The energy deposition depends on the detector thickness, which is measured to a quoted accuracy of $\pm 2 \mu \mathrm{m}$ by the manufacturer 1 , and the well-determined direction of the muon relative to the detector plane. The detectors were biased to full depletion, thus providing uniform charge collection through the entire thickness.

For alpha sources, the non-linear terms and other corrections are important and the energy calibration is described by

$$
E_{p}=E_{\text {det }}+\Delta E_{s}+\Delta E_{a d c}+\Delta E_{n l}\left(E_{p}\right)
$$

where $E_{p}$ and $E_{\text {det }}$ are the true and detected energies respectively, $\Delta E_{s}$ is the energy lost through the source thickness, $\Delta E_{a d c}$ is the non-linearity of the ADC as determined by a precision pulser, and $\Delta E_{n l}$ stands for the non-linear response corrections. The detected energy is assumed to be linearly proportional to the channel number $x, E_{\text {det }}=a_{0}+a_{1} x$, with $a_{0}$ and $a_{1}$ being the parameters of a linear fit. The calculation of the different terms is described below.

The ${ }^{244} \mathrm{Cm}$ and ${ }^{148} \mathrm{Gd}$ calibration sources which were mounted inside the BPT during the ${ }^{8} \mathrm{~B}$ run are thick sources produced by drying a droplet of solution containing the activity onto a stainless steel backing. These sources have since been replaced with commercial electroplated spectroscopy grade thin sources with peak widths of $20 \mathrm{keV}$ FWHM and will decrease the uncertainty of future calibrations. The energy loss $\Delta E_{s}$ due to source thickness was determined by measuring the sources against ultra-thin alpha sources produced using mass-separated radioactive beams [20]. These sources are effectively carrier-free and are used to investigate the depth profiles of the thick and thin sources. Figure 5 shows a comparison between the ${ }^{244} \mathrm{Cm}$

\footnotetext{
${ }^{1}$ Micron Semiconductor Ltd., Lancing, UK.
}

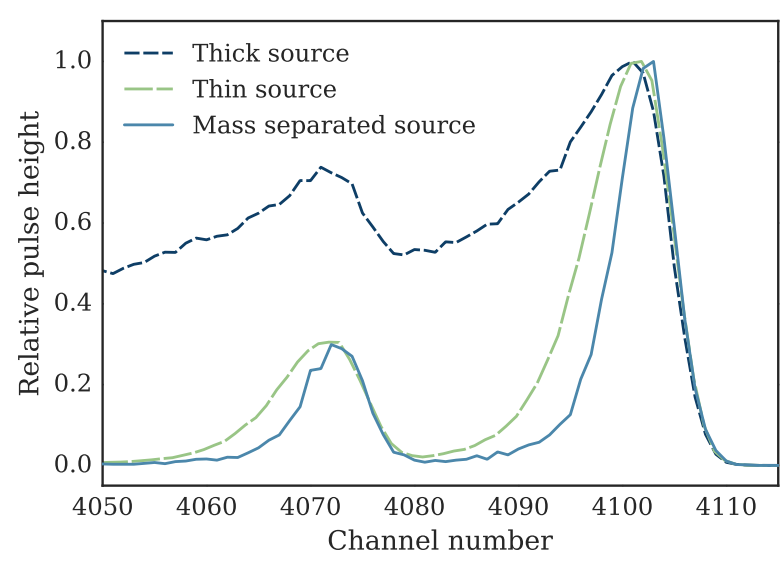

Figure 5: Spectra of the two dominant peaks of ${ }^{244} \mathrm{Cm}$ as measured using a PIPS detector for the sources used in this work. The sources used were a thick source, made by a drying process and used for the calibrations in this paper, a thin commercial spectroscopic source, and a carrier-free source made by implantation of a mass-separated beam.

spectra from the different sources recorded using a 300$\mu \mathrm{m}$-thick, 25- $\mathrm{mm}^{2}$-area passivated implanted planar silicon (PIPS) detector. The long tails of the thick source are an indication of a depth profile for the activity that most probably formed during the drying process, resulting in thicker edges that appear visually different. This was confirmed by measuring a source with a mask over its edges which resulted in the elimination of the long tails. Due to the complex profile of the sources, the values of $\Delta E_{s}$ are calculated by comparing the measurements to a response function model described below.

A depth profile, composed of a combination of thin (inner) and thick (outer) regions was simulated using TRIM 24] to match the shape of the measured thick source spectrum. It is also clear from simulations that the assumption of source uniformity for this source is indeed unjustified and could lead to false determination of $\Delta E_{s}$ by more than $10 \mathrm{keV}$. The good agreement between the response function simulation and measurements performed with the PIPS detector near the alpha-particle energy peak increases our confidence in the validity of the model for the DSSD performance. Due to the complex lineshape of the peak from the thick source, the half-width-half-maximum (HWHM) of the peak edge is used for the energy cali$y_{5}$ ration and is related to the edge and peak of the massseparated source using the simulation and taking into account the different characteristics of both the PIPS and DSSD detectors. The energy losses due to the source thickness $\Delta E_{s}$ are determined to be 7.0 and $8.5 \mathrm{keV}$ for ${ }^{148} \mathrm{Gd}$ and ${ }^{244} \mathrm{Cm}$, respectively.

The (non)linearity of the ADC, $\Delta E_{a d c}$ was determined to be typically less than $5 \mathrm{keV}$ over the entire energy range using a precision pulser.

The non-linear correction term, $\Delta E_{n l}$, is a collection of three effects: (1) the energy loss in the detector dead layer, 
$\Delta E_{d l}$, (2) the non-ionizing energy loss, $\Delta E_{n}$, due to effects such as nuclear reactions or lattice vibrations, and (3) what generally is known as the pulse height defect [21. This is the result of local energy deposition by the interaction of heavy ions that decreases the energy required for creation of an electron-hole pair, effectively creating higher charge for a given energy. This effect is dominant for low-Z ions such as alphas and results in a non-linear increase of the recorded energy. For higher-Z ions still, the higher energy loss liberates sufficient charge locally to shield the applied electric field inside the detector which results in losses in charge collection. This effect reduces the detected energy for higher-Z ions but is not significant for alpha particles.

Under the assumption that $\left(\Delta E_{d l}+\Delta E_{n}\right) / E_{p}<<1$ and following the derivation of Munnik et al [22, $\Delta E_{n l}$ is expanded to

$$
\begin{aligned}
\Delta E_{n l}\left(E_{p}\right) & \simeq \Delta E_{d l}\left(1+\frac{k}{\epsilon_{0}} S\left(E_{p}\right)\right) \\
& +\Delta E_{n}\left(1+\frac{k}{\epsilon_{0}} S\left(E_{p}\right)\right) \\
& -\frac{k}{\epsilon_{0}} \int_{0}^{E_{p}} S(E) d E .
\end{aligned}
$$

where $\epsilon_{0}$ is the electron-hole pair creation energy in silicon $(3.67 \mathrm{eV}), S(E)$ is the stopping power of alphas in silicon and $k$ is a factor, determined by Bauer and Bortels to be $2.8(3) \cdot 10^{-4} \mathrm{~nm} /$ electron-hole pair 23 .

The detector dead-layer and non-ionizing energy losses are also calculated with a detailed Monte-Carlo simulation using TRIM [24] to determine both the average energy loss and the distribution resulting from straggling or phononcreation processes. While dead-layer straggling produces a distribution that is nearly symmetric, the distribution of non-ionizing energy losses is skewed with a tail that extends $\sim 100 \mathrm{keV}$ below the peak. Figure 6 shows the simulated non-ionizing energy loss $\Delta E_{n}$ as a function of the alpha energy. The mean of the distributions is in agreement with previous calculations [4, 25.

By simulating the energy-loss distribution for a specific source depth profile and dead-layer width and by convoluting with the electronic noise, Fano factor, and the asymmetrical non-ionizing distributions, the response function of any alpha source can be simulated and reconstructed [26. Results of TRIM simulations and PIPS measurements of ${ }^{148} \mathrm{Gd}$ measured with the thick and mass-sperated sources are shown in Fig. 7

After taking into account all the aforementioned nonlinear terms, the energy calibration is then performed by fitting a linear function to the three $E_{\text {det }}$ points. Since all non-linear effects are removed, they should fall on a straight line with a slope corresponding to $\epsilon_{0}=3.67 \mathrm{eV}$ energy deposited per pair for both muons and alpha particles. Table 1 summarizes the energy parameters and uncertainties calculated for the calibration of one of the front strips of the Top detector based on the analysis of

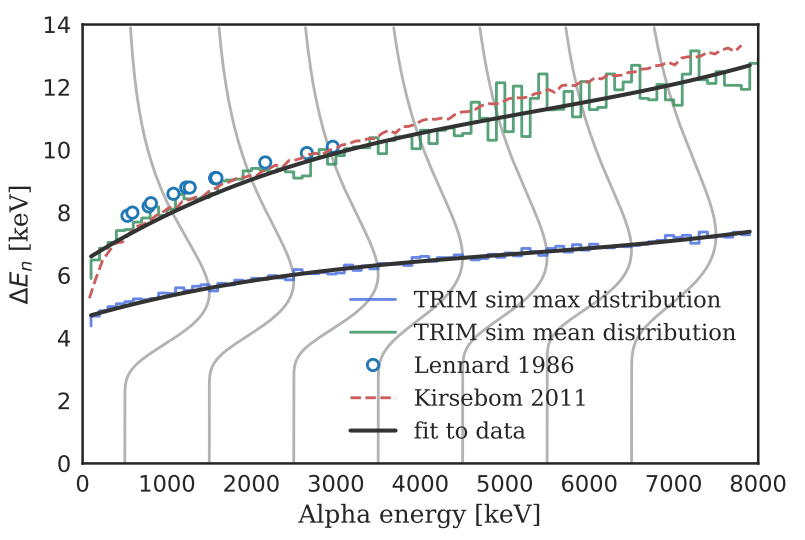

Figure 6: Non-ionizing energy loss calculated using TRIM for alpha particles in silicon. Detailed simulations of the energy loss result in the skewed distributions illustrated as vertical curves in the figure background. The maximum (most probable value) and mean of the distribution are shown as step-lines with a spline fit imposed. Previous calculations of Lennard et al. 25] and Kirsebom et al. 44 are in good agreement with the calculated mean. In the current analysis the whole distribution is used while $\Delta E_{n}$ is defined as the maximum energy of the non-ionizing distributions.

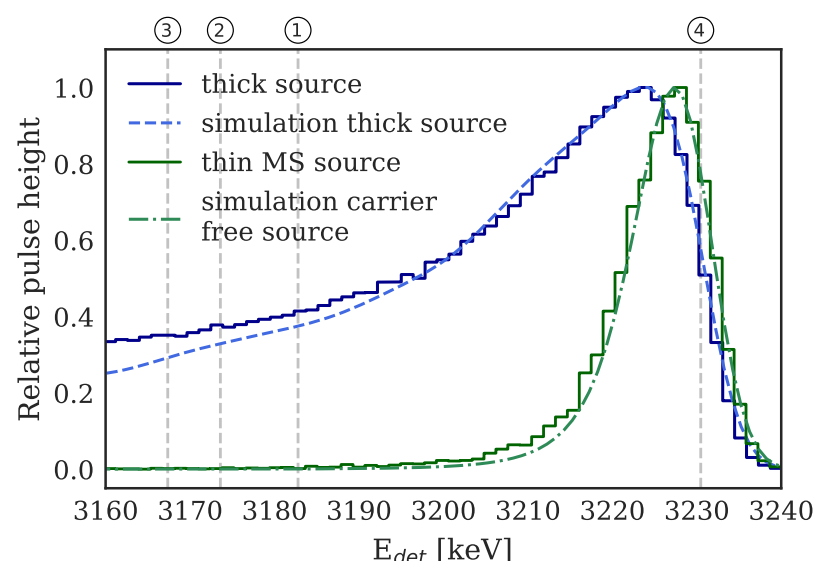

Figure 7: Detected energy spectrum of the ${ }^{148} \mathrm{Gd}$ sources used in this work as measured using the PIPS detector and simulated by TRIM. The only parameter changed in the simulations is the source depth profile. The energy on the $\mathrm{x}$ axis is the detected energy, vertical dashed-lines indicate the true (1), dead layer corrected (2), nonionizing corrected (3), and pulse-height defect corrected (4) energies. The true energy (1)corresponds to the energy deposited by a minimum ionizing particle in the detector that would yield the observed pulse height. The energy difference between the HWHM of the thick source distribution and the effective energy of line (4) is defined as $\Delta E_{s}$ for this specific source and is a function of the electronic noise. 
Top-Bottom coincidences. Here, $E_{\text {det }}$ stands for the simulated energy of the muons and the detected energies of the alpha sources following non-linear corrections. The values quoted as the pulse heights are the peak most probable value $(m p v)$ and the $H W H M$ peak edge for the case of muons and alphas respectively. The total uncertainties are calculated by taking the square-root of the sum of the squared uncertainties.

The dead-layer uncertainties are taken as an upper limit of $1 \mathrm{keV}$, in accordance to the accuracy of recent dead-layer measurements [7. The uncertainties due to non-ionizing processes are taken as $15 \%$ of the average value, as suggested in Ref. 27. The pulse-height defect uncertainty is taken as $5 \%$, which is the upper limit of the uncertainty quoted for PHD in alphas in Ref. [27. The uncertainty of the Geant4 simulation of the muon energy deposition is estimated to be $1.5 \%$ [28]. Lastly, the thicknesses of the Top and Bottom detectors, as measured by the detector manufacturer, are both $1033 \pm 2 \mu \mathrm{m}$, which translates to an uncertainty in the muon energy deposition of less than $1 \mathrm{keV}$.

Table 1: Data points and associated uncertainties used for the calibration of a typical front strip of the Top detector (top table), uncertainty budget (middle table) and a comparison between the fit coefficients obtained from using both alpha and muon data compared to a calibration based on the alpha sources alone.

\begin{tabular}{lrrc}
\hline & Muons & ${ }^{148} \mathrm{Gd}$ & ${ }^{244} \mathrm{Cm}$ \\
\hline$E_{p}, \mathrm{keV}$ & & 3182.78 & 5804.82 \\
$E_{\text {det }}, \mathrm{keV}$ & 319.88 & 3225.85 & 5888.51 \\
Pulse height, Channels & 183.28 & 1638.40 & 2971.16 \\
\hline Errors, keV & & & \\
\hline Peak fit & 4.96 & 0.72 & 0.55 \\
Dead-layer & & 1.0 & 1.0 \\
Non-ionizing & & 1.8 & 2.1 \\
Pulse-height defect & & 3.0 & 4.6 \\
Source thickness & & 3.0 & 3.0 \\
ADC non-linearity & 0.31 & 0.27 & 0.38 \\
Detector thickness & 0.61 & & \\
Total error & 5.01 & 4.78 & 6.00 \\
\hline Calibration & intercept & slope & \\
\hline Only alpha sources & $-47.41(10)$ & $1.998(5)$ & \\
Alpha sources + muons & $-46.44(5)$ & $1.997(2)$ & \\
\hline
\end{tabular}

Figure 8 shows the energy residuals of the calibration (data - calibration). The $1 \sigma$ uncertainty band is calculated by bootstrapping the calibration curve with the uncertainties of the polynomial fit at the weighted average energy. The advantage of using the additional information from the muons can be seen from the reduction in the uncertainty of the energy calibration. At the $1-2 \mathrm{MeV}$ energy region, which is important for studies of ${ }^{8} \mathrm{Li}$ and ${ }^{8} \mathrm{~B} \beta$ delayed $\alpha$ emission, the uncertainty obtained by including the muon data is $3 \mathrm{keV}$ on average over all strips, compared to $7 \mathrm{keV}$ without it. The calibrations agree within their 1-

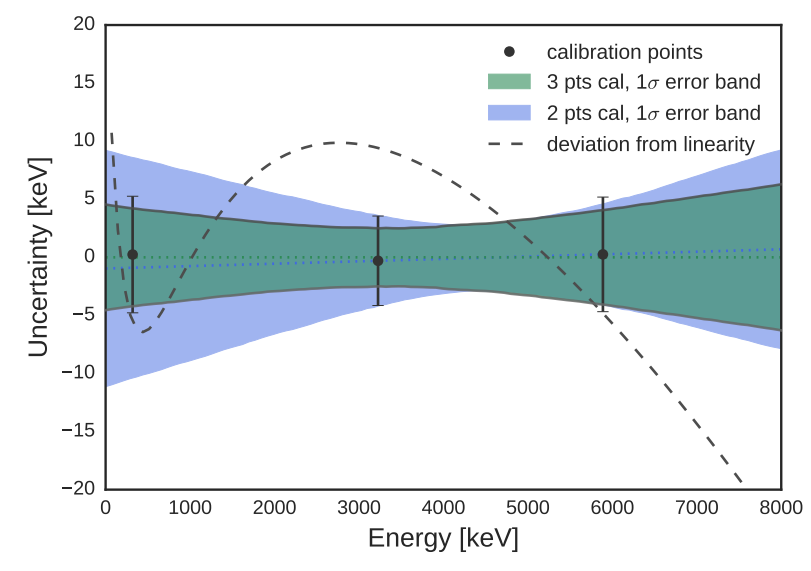

Figure 8: Residuals (data - calibration) and uncertainty bands of the calibration for a typical strip of the Top detector. The solid circles, from left to right, show the residuals of the muon peak and ${ }^{148} \mathrm{Gd}$ and ${ }^{244} \mathrm{Cm}$ alpha sources. The $1 \sigma$ uncertainty band is represented by a green envelope. The calibration and uncertainty using only the two alpha source points is shown as a blue envelope. The dashed curve shows the difference in the calibration if a simple linear approximation is used for the pulse-height defect.

$\sigma$ uncertainty bands over the entire energy region. While this agreement is not a measure of a calibration quality, it does strengthen our confidence in the new calibration method. As discussed above, the influence of the pulseheight defect on the detector response has a significant effect in the energy range of study. A linear approximation of this effect may lead to systematic errors of 10-20 $\mathrm{keV}$ as indicated by the dashed line which shows the results of a calibration achieved by assuming that $\Delta E_{n l}$ is linear.

\section{Discussion and summary}

In the BPT detection system, a calibration with muons can be performed with reasonable statistics within 24 hours. The identification of muon events will also benefit from the recent installation of thick plastic scintillator detectors behind the DSSDs. The muons would trigger a coincidence between two plastics and two DSSDs, allowing a clear identification of these events.

The calibration process shown in this paper is based on coincidences from the Top and Bottom DSSDs, which naturally have better statistics, due to the predominantly downward direction of incoming muons. The Right-Left DSSD pair has about one third the number of muon events as the Top-Bottom pair, potentially requiring longer measurements to allow precise calibration of those detectors. However, by considering coincidences of 90-degree detector pairs (e.g. Top with Left or Right), higher statistics can be achieved. It should be noted that for these detector pairs the energy of the muon peak is shifted towards higher energies due to the larger muon impinging angles. This phenomenon could be utilized to calibrate over sev- 
eral energies in the $0.3-1 \mathrm{MeV}$ range by sorting according to the incident angle. By comparing the data from all possible coincidences between detectors to simulations, a precise calibration of all four DSSDs is possible.

This paper demonstrates a method to take advantage of the continuously-available cosmic-ray muon flux to provide an additional calibration point to constrain the energy response of DSSDs below $\sim 1 \mathrm{MeV}$. The measured muon spectra are compared to detailed Monte-Carlo simulations to provide data point to the calibration. The inclusion of muons improves the energy calibration at low energies. The better energy-calibration achieved using the method proposed in this article could be used to lower one of the principle sources of uncertainty in many of the measurements conducted with the BPT or other equivalent systems.

\section{Acknowledgment}

The authors would like to thank I. Ahmad and J. Greene of Argonne National Laboratory for their help and fruitful discussions in the characterization of alpha sources. This work was supported by the U.S. Department of Energy, Office of Nuclear Physics, under Contracts No. DE-AC0206CH11357 (Argonne National Laboratory) and DE-AC5207NA27344 (Lawrence Livermore National Laboratory), NSERC Application Number SAPPJ-2015-00034 (CPI 11991 and NSF Grants PHY-1419765 and PHY-1430152. T.Y.H would also like to thank the support from the Pazi foundation.

\section{References}

[1] J. N. Bahcall, E. Lisi, D. E. Alburger, L. de Braeckeleer, et al., Phys. Rev. C 54 (1996) 411-422. arXiv:nucl-th/9601044 doi : 10.1103/PhysRevC.54.411

[2] W. T. Winter, S. J. Freedman, Phys. Rev. C 73 (2) (2006) 025503. doi:10.1103/PhysRevC.73.025503.

[3] M. Bhattacharya, E. G. Adelberger, H. E. Swanson, Phys. Rev. C 73 (5) (2006) 055802. doi:10.1103/PhysRevC.73.055802.

[4] O. S. Kirsebom, S. Hyldegaard, M. Alcorta, M. J. G. Borge, et al., Phys. Rev. C 83 (6) (2011) 065802. doi:10.1103/ PhysRevC.83.065802.

[5] T. Roger, J. Büscher, B. Bastin, O. S. Kirsebom, et al., Phys. Rev. Lett. 108 (2012) 162502. doi:10.1103/PhysRevLett.108. 162502

[6] G. Li, R. Segel, N. D. Scielzo, P. F. Bertone, et al., Physical Review Letters 110 (9) (2013) 092502. doi:10.1103/PhysRevLett. 110.092502

[7] M. G. Sternberg, R. Segel, N. D. Scielzo, G. Savard, et al., Phys. Rev. Lett. 182501 (October) (2015) 1-6. doi:10.1103/ PhysRevLett.115.182501.

[8] D. Barney, W. Bialas, P. Kokkas, N. Manthos, others., Nucl. Instruments Methods Phys. Res. A 564 (1) (2006) 126-133. doi : 10.1016/j.nima.2006.03.031

[9] N. D. Scielzo, G. Li, M. G. Sternberg, G. Savard, et al., Nucl. Instruments Methods Phys. Res. A 681 (2012) 94-100. doi: 10.1016/j.nima.2012.04.035

[10] R. M. Yee, N. D. Scielzo, P. F. Bertone, F. Buchinger, et al., Phys. Rev. Lett. 110 (2013) 092501. doi:10.1103/PhysRevLett. 110.092501
[11] N. D. Scielzo, R. M. Yee, P. F. Bertone, F. Buchinger, et al., Nuclear Data Sheets 120 (2014) 70-73. doi:10.1016/j.nds. 2014.07.009

[12] M. G. Sternberg, Ph.D. thesis, The University of Chicago (2013).

[13] D. E. Groom, N. V. Mokhov, S. I. Striganov, At. Data Nucl. Data Tables 78 (2) (2001) 183-356. doi:10.1006/adnd.2001. 0861

[14] S. Agostinelli, J. Allison, K. Amako, J. o. Apostolakis, Nucl. Instruments Methods Phys. Res. A 506 (3) (2003) 250-303. doi: 10.1016/S0168-9002(03)01368-8

[15] K. Amako, S. Guatelli, V. Ivanchenko, M. Maire, others., Nuclear Science, IEEE Transactions on 52 (4) (2005) 910-918. doi:10.1109/TNS.2005.852691

[16] C. Hagmann, D. Lange, D. Wright, in: Nuclear Science Symposium Conference Record, 2007. NSS '07. IEEE, Vol. 2, 2007, pp. 1143-1146. doi:10.1109/NSSMIC.2007.4437209

[17] M. Hohlmann, P. Ford, K. Gnanvo, J. Helsby, et al., Nuclear Science, IEEE Transactions on 56 (3) (2009) 1356-1363. doi: 10.1109/TNS.2009.2016197

[18] E. Aguayo, R. Kouzes, A. Ankney, J. Orrell, et al., Pacific Northwest National Laboratory PNNL-20693 (2011) .

[19] L. Landau, J.Phys.(USSR) 8 (1944) 201-205.

[20] I. Ahmad, J. P. Greene, F. G. Kondev, S. Zhu, Phys. Rev. C 91 (4) (2015) 044310. doi:10.1103/PhysRevC.91.044310

[21] H. Schmitt, W. Gibson, J. Neiler, F. Walter, et al., in: International Atomic Energy Agency, Symposium on Physics and Chemistry of Fission, 22-26 Mar 1965, IAEA-SM-60/40, 1965, pp. 531-545.

[22] F. Munnik, A. J. M. Plompen, J. Räisänen, U. Wätjen, Nucl. Instruments Methods Phys. Res. B 119 (4) (1996) 445-451. doi: 10.1016/S0168-583X (96) 00468-5

[23] P. Bauer, G. Bortels, Nucl. Instruments Methods Phys. Res. A 299 (1990) 205-209.

[24] J. F. Ziegler, M. D. Ziegler, J. P. Biersack, Nucl. Instruments Methods Phys. Res. B 268 (11-12) (2010) 1818-1823. doi:10. 1016/j.nimb.2010.02.091

[25] W. N. Lennard, H. Geissel, K. B. Winterbon, D. Phillips, et al., Nucl. Instruments Methods Phys. Res. A 248 (1986) 454-460. doi:10.1016/0168-9002(86)91033-8

[26] E. Steinbauer, P. Bauer, M. Geretschläger, G. Bortels, et al., Nuclear Instruments and Methods B 85 (1994) 642-649. doi: 10.1016/0168-583X (94) 95898-X

[27] W. N. Lennard, G. R. Massoumi, Nuclear Instruments and Methods B 48 (1990) 47-50. doi:10.1016/0168-583X(90) 90070-B

[28] A. Schälicke, A. Bagulya, Ø. Dale, F. Dupertuis, et al., Journal of Physics Conference Series 331 (3) (2011) 032029. doi:10. 1088/1742-6596/331/3/032029 